\title{
Convergence analysis of the semi-implicit Euler method for abstract evolution equations
}

\author{
Renato Spigler ${ }^{1}$ and Marco Vianello ${ }^{2}$ \\ Institute for Mathematics and its Applications, \\ University of Minnesota, \\ 514 Vincent Hall, \\ 206 Church Street S.E., \\ Minneapolis, MN 55455-0436.
}

\begin{abstract}
The semi-implicit Euler discretization method is studied for abstract evolution equations in a Hilbert space $\mathcal{H}$, like $\dot{u}=f(t, u, u), t \in(0, T], u(0)=u_{0}$, where $f(t, \cdot, v)$ is one-sided Lipschitz and $\mathcal{R}(I-h f(t, \cdot, v))=\mathcal{H}$ for $h>0$ sufficiently small, and $f(t, u, \cdot)$ is Lipschitz-continuous. Extension to Banach spaces is then pointed out. Ordinary and partial, differential and integro-differential equations or systems are included. For instance, $\dot{u}=A(t, u)+B(t, u)$, where $A(t, \cdot)$ is [strongly] dissipative and maximal, and $B(t, \cdot)$ is Lipschitz-continuous, fall into the previous class. The scheme is $u_{n+1}=u_{n}+\Delta t f\left((n+1) \Delta t, u_{n+1}, u_{n}\right)$, $n=0,1, \ldots, N-1$, where $\Delta t:=T / N$. Two main computational advantages with respect to fully implicit methods are: (a) linearization of semilinear problems, and (b) decoupling of systems into lower-dimensional (stationary) subsystems, at each time step. In the latter case, parallelization becomes possible. A full error analysis is performed: consistency and stability are estabilished, and precise convergence estimates are obtained. Several applications, including reaction-diffusion and hyperbolic systems, are finally given.
\end{abstract}

\footnotetext{
${ }^{1}$ Permanent address: Dipartimento di Metodi e Modelli Matematici per le Scienze Applicate, Universitá di Padova, Via Belzoni 7, 35131 Padova, Italy.

${ }^{2}$ Dipartimento di Matematica Pura e Applicata, Universitá di Padova, Via Belzoni 7, 35131 Padova, Italy.
} 


\section{Introduction.}

In this paper we are concerned with the numerical treatment of abstract nonlinear evolution problems of a rather general class that includes as a special case

$$
\dot{u}=A(t, u)+B(t, u), \quad 0<t \leq T ; u(0)=u_{0} .
$$

Here $A(t, \cdot):\left(D_{t} \subseteq \mathcal{H}\right) \rightarrow \mathcal{H}, t>0$, denotes a [strongly] dissipative and maximal operator [33], in general nonlinear, $\mathcal{H}$ being a real or complex Hilbert space, and $B(t, \cdot):(D \subseteq \mathcal{H}) \rightarrow \mathcal{H}$ is a uniformly Lipschitz continuous operator, with Lipschitz constant $K>0, \bigcup_{t>0} D_{t} \subseteq D \subseteq \mathcal{H}$. The method consists in reducing the numerical solution of (1) to the solution of a sequence of stationary problems, arising from a semi-implicit time discretization of (1), as

$u_{n+1}=u_{n}+h A\left((n+1) h, u_{n+1}\right)+h B\left((n+1) h, u_{n}\right), \quad n=0,1, \ldots, N-1, \quad h:=T / N$,

$h>0$ denoting the time discretization step. Such problems are linear when $A(t, \cdot) \equiv$ $A(t) \cdot$ is a linear operator: This is the case, for instance, when

$$
A(t, \cdot)=\sum_{i, j} a_{i j}(x, t) \frac{\partial^{2}}{\partial x_{i} \partial x_{j}}+\sum_{i} b_{i}(x, t) \frac{\partial}{\partial x_{i}}+c(x, t)
$$

is a linear elliptic operator, i.e. when (1) is a semilinear partial differential equation of parabolic type. Existence, uniqueness and regularity of solutions to equations like (1) have been studied by several authors (see [20, 23], e.g.). Treating by the semiimplicit Euler method (2) an equation like (1) may be advantageous, in that it leads to linearization, in case of semilinear equations; to decoupling into lower-dimensional stationary subsystems in case that (1) represents a system of ordinary [28, 29] or partial differential equations; or to equations characterized by local rather than nonlocal (e.g. integral) operators. The latter is typical of linear partial integro-differential equations (where integration is over the space domain), in which case the method leads, at each time step after space-discretization, to sparse or band-structured, instead of full, systems. When (2) decouples into subsystems, the ensuing computational advantage is clear, as parallel implementation becomes feasible (see [28, 29], e.g., for systems of ordinary differential equations). These observations show the importance of adopting the semi-implicit Euler method, while only the fully implicit method seems to have been widely used so far (see [19, 22, 24, 26] and references therein), at least in the infinite-dimensional case. Other semi-implicit methods for special evolutionary PDE problems do appear in the literature. Concerning nonlinear parabolic equations, one should mention [30], where, however, the equation was first discretized in space by a Galerkin finite element scheme, thus obtaining a linear system of ODEs. In another 
direction, semi-implicit finite difference discretizations (both in space and in time) have been used to solve shallow water equations, see [6].

In this paper, we are actually able to study stability and consistency (and thus convergence, $\mathrm{cf}$. [7]) of certain semi-implicit schemes for (abstract) evolution problems in Hilbert space, like

$$
\dot{u}=f(t, u, u), \quad 0<t \leq T ; u(0)=u_{0},
$$

where $f$ has the (uniform) Lipschitz property with respect to the scalar product (one-sided Lipschitz condition) in the second argument, and is (uniformly) Lipschitzcontinuous in the third. Equation (1) above is a special case of (3). As the consistency analysis is based on some regularity conditions, throughout the paper we assume, besides existence and uniqueness of a strong solution $u$ to (3), that $u \in C^{1}[0, T]$, or $\dot{u} \in B V[0, T]$ (and $u \in A C[0, T]$ ).

The procedure we follow to analyze the implicit Euler method for (3) is based on the general theory of convergence for numerical methods developed by B. Chartres

and R. Stepleman [7, 8]. Such a theory has the feature of allowing to take into account any kind of "perturbations" (e.g. truncation as well as round-off errors), at the same time. In Section 2, we review briefly such a theory, and in Section 3 the semi-implicit discretization scheme is presented. Stability and consistency are here estabilished, along with precise convergence estimates. Extension to evolutionary problems in Banach spaces is then pointed out. In Section 4, several applications are given, which include equations and systems, differential and integro-differential, of the reaction-diffusion type, as well as semilinear hyperbolic equations and systems. In Section 5, finally, we collect some general remarks.

\section{A brief account of the Chartres-Stepleman the- ory.}

In [7], B. Chartres and R. Stepleman introduced an abstract framework to analyze general numerical methods, see also [8]. Their approach, which originated by earlier basic ideas of I. Babuska et al. [2], is quite flexible and general. A "numerical method" is defined as a sextuple

$$
\left\{X, Y, F, H,\left\{F_{h}\right\},\left\{E_{h}\right\}\right\},
$$

$X$ denoting the "data space", $Y$ the "solution space", $H$ the "discretization parameters set". $F: X \rightarrow Y$ is the mapping that associates the data to the solution of the underlying problem. Usually, $Y$ is a linear normed space, equipped with the norm $\|\cdot\|_{Y}$, but it could be merely a metric space or even a topological vector space. $H$ is a set of "discretization steps" where a convergence to 0 is defined, though $0 \notin H$. 
The approximating procedure is described by means of the family of operators $\left\{F_{h}\right\}_{h \in H}, F_{h}: X \times E_{h} \rightarrow Y,(x, e) \mapsto F_{h}(x, e)$, where $\left\{E_{h}\right\}_{h \in H}$ denotes a family of "perturbation spaces". The space $E_{h}$ contains, for each $h \in H$, an element denoted by 0 , and a topology is given in $E_{h}$ by means of a function $\phi_{h}: E_{h} \rightarrow R_{0}^{+}, \phi_{h}(0)=0$. A basis of neighborhoods of 0 is defined as $N_{0}(\delta):=\left\{e \in E_{h}: \phi_{h}(e)<\delta\right\}, \delta>0$. The function $\phi_{h}(e)$ measures, for every fixed $h$, the perturbation $e$. In most cases, $E_{h}$ is a linear normed space, and $\phi_{h}$ is a norm on $E_{h}$.

The method (4) is termed convergent at $x \in X$ if

$$
\left\|F_{h}(x, e)-F(x)\right\|_{Y} \rightarrow 0, \text { as } h \rightarrow 0, \quad \phi_{h}(e) \rightarrow 0,
$$

and is termed stable at $x \in X$ if

$$
\| F_{h}(x, e)-F_{h}\left(x, 0 \|_{Y} \rightarrow 0, \text { as } h \rightarrow 0, \phi_{h}(e) \rightarrow 0 .\right.
$$

Moreover, it is said to be consistent if, for every $h \in H$, there exists a special perturbation, say $\hat{e}_{h}(x)$, depending in general on $x$ and $h$, such that

$$
\left\|F_{h}\left(x, \hat{e}_{h}(x)\right)-F(x)\right\|_{Y} \rightarrow 0, \text { as } h \rightarrow 0,
$$

with

$$
\phi_{h}\left(\hat{e}_{h}(x)\right) \rightarrow 0, \text { as } h \rightarrow 0 .
$$

It is then easy to prove (cf. [7]) that a general form of the celebrated Lax Equivalence Theorem [17] holds: A numerical method (defined as in (4)) is convergent (as in (5)) if and only if it is stable (as in (6)) and consistent (as in (7), (8)).

It is worth noting that the present definition of convergence is more general than the usual one. In fact, in the literature it is often required only that $F_{h}(x, 0) \rightarrow F(x)$ as $h \rightarrow 0$, i.e. that the "exact" (unperturbed) scheme converges to the solution of the original problem. The notion of convergence in (5) takes into account, however, the presence of unavoidable perturbations. Therefore, the usual convergence concept can be seen as a special case of consistency (taking $\hat{e}_{h}(x) \equiv 0$ ), and is implied by stability and consistency as above, in view of Lax theorem.

\section{Stability and consistency of the semi-implicit Eu- ler method.}

In this Section we prove stability ( $(3.1)$ and consistency ( $\S 3.2)$ according to the definitions given in Section 2, for a semi-implicit Euler scheme applied to

$$
\dot{u}=f(t, u, u), \quad 0<t \leq T ; u(0)=u_{0} .
$$


Here $f(t, \cdot, v):\left(D_{t} \subseteq \mathcal{H}\right) \rightarrow \mathcal{H}, \mathcal{H}$ being a real Hilbert space, $t>0, v \in D$, with $\bigcup_{t>0} D_{t} \subseteq D \subseteq \mathcal{H}$, satisfies a Lipschitz condition with respect to the scalar product $\langle\cdot, \cdot\rangle$ in $\mathcal{H}$ (one-sided Lipschitz condition), i.e., for every fixed $t \in(0, T]$ and $v \in D$,

$$
\left\langle f\left(t, u_{1}, v\right)-f\left(t, u_{2}, v\right), u_{1}-u_{2}\right\rangle \leq K_{1}\left\|u_{1}-u_{2}\right\|^{2}, \forall u_{1}, u_{2} \in D_{t},
$$

with a constant $K_{1} \in R$, and $f(t, u, \cdot):(D \subseteq \mathcal{H}) \rightarrow \mathcal{H}, t>0, u \in D_{t}$, is uniformly Lipschitz continuous in the classical sense, with a constant $K_{2}>0$

$$
\left\|f\left(t, u, v_{1}\right)-f\left(t, u, v_{2}\right)\right\| \leq K_{2}\left\|v_{1}-v_{2}\right\|
$$

Moreover, we assume that for each $h>0$ sufficiently small $\mathcal{R}(I-h f(t, \cdot, v))=\mathcal{H}$, namely that the equation

$$
u=h f(t, u, v)+b
$$

has a unique solution $u$ in $D_{t}$, for each fixed $t \in(0, T], v \in D, b \in \mathcal{H}$, and $h>0$ sufficiently small. When $f$ is dissipative in $u\left(K_{1}=0\right)$, the solvability of $(12)$ is equivalent to that of the same equation with $h=1$, since the operator $f(t, \cdot, v)$ is maximal in this case; cf. [4, 20, 33].

In particular, $f(t, u, v)=A(t, u)+B(t, v)$, where $A(t, \cdot)$ is dissipative, or strongly dissipative $\left(K_{1}<0\right)$ and maximal [33], and $B(t, \cdot)$ is Lipschitz continuous (cf. $\S 1$ ), as it occurs in typical cases arising within the context of parabolic differential or integrodifferential equations. In these cases, equation (12) is an elliptic partial differential or integrodifferential equation, and its solvability must be explicitly required.

By a solution to $(9)$ we mean a function $u \in C^{0}([0, T] ; \mathcal{H})$, such that $u(t) \in D_{t}$ for every $t \in(0, T], T$ being a fixed positive number, strongly differentiable in $(0, T]$, satisfying (9). Under assumptions (10), (11), uniqueness can be estabilished in a standard way by using differential inequalities (cf. [16], e.g.), $f(t, u, u)$ being onesided Lipschitz as a function of $u$, with constant $K_{1}+K_{2}$. We assume that further hypotheses are made, when necessary, ensuring existence of solutions $u \in C^{1}[0, T]$, or $u \in A C[0, T], \dot{u} \in B V[0, T]$ (cf. [13, Ch.3] concerning the definitions of $A C$ and $B V$ functions in the abstract context). Indeed, our algorithm is able to approximate solutions in these classes in the $C^{0}$-norm.

According to the formalism introduced in Section 2, here we take for $X$ the set of all initial data $u_{0}=x \in D$ for which a solution with the properties above exists (necessarily $\left.u_{0} \in \operatorname{cl}\left(\bigcup_{t>0} D_{t}\right)\right) ; Y=C^{0}([0, T] ; \mathcal{H})$, since we shall approximate the solution $u$ by linear interpolation; $F$ maps $u_{0}$ into the unique solution $u$ to (9); $H=\left\{h=T / N, N \in N^{+}\right\}$. The approximating procedure is based on the following (ideal) iterative algorithm: For $n=0,1,2, \ldots, N$, set $t_{n}=n h$, and

$$
u_{n+1}=u_{n}+h f\left(t_{n+1}, u_{n+1}, u_{n}\right) \text {. }
$$


As we have to take into account all unavoidable errors introduced on each step, we consider the perturbed scheme

$$
\begin{gathered}
v_{n+1}=\tilde{u}_{n}+h f\left(t_{n+1}, v_{n+1}, \tilde{u}_{n}\right)+\delta_{n+1}, \\
\tilde{u}_{n+1}=v_{n+1}+\sigma_{n+1}, \quad \tilde{u}_{n+1} \in D .
\end{gathered}
$$

Here $\delta_{0}=\tilde{u}_{0}-u_{0}$ represents the error on the initial data, $\tilde{u}_{0} \in D$, and $\sigma_{n} \in \mathcal{H}$, $n=1,2, \ldots, N-1$, is the error made in solving approximately the first equation in (14). The perturbation term $\sigma_{n+1}$ cannot, in general, be absorbed in $\delta_{n+1}$, as it is possible in the case of ordinary differential equations, where $f$ is Lipschitz continuous (in the classical sense) also with respect to its second argument. The scheme (14) is well-defined since, for every $n$, the first equation in (14) has a unique solution $v_{n+1}$ in view of (10), (11) (when $K_{1}>0$, uniqueness is guaranteed at least for $h$ sufficiently small, which fact, in turn, modifies the set $H$ above). From the scheme in (14) it is clear that one should require explicitely that $\tilde{u}_{n} \in D$ at each time step. In other words, this means that the method to be used for solving the first equation in (14) must give an approximate solution, $\tilde{u}_{n+1}$, in $D$.

Finally, the "perturbation space", $E_{h}$ (cf. $\S 2$ ), consists of all admissible "perturbations vectors" $(\delta, \sigma)=\left(\delta_{0}, \delta_{1}, \ldots, \delta_{N} ; \sigma_{1}, \sigma_{2}, \ldots, \sigma_{N}\right)$ described above, and thus $E_{h} \subseteq$

$\mathcal{H}^{2 N+1}$. The approximating mapping, $F_{h}$ (cf. $\S 2$ ), is taken to be the linear interpolant among the values $\tilde{u}_{n}, n=0,1,2, \ldots, N$. The function $\phi_{h}$ (cf. $\S 2$ ) that introduces the topology on $E_{h}$, will be defined in $\S 3.1$.

\subsection{Stability of the semi-implicit Euler method.}

From (13), (14), we obtain

$$
\tilde{u}_{n+1}-u_{n+1}=v_{n+1}-u_{n+1}+\sigma_{n+1}
$$

and

$$
\begin{aligned}
v_{n+1}-u_{n+1} & =\tilde{u}_{n}-u_{n}+h\left[f\left(t_{n+1}, v_{n+1}, \tilde{u}_{n}\right)-f\left(t_{n+1}, u_{n+1}, u_{n}\right)\right]+\delta_{n+1} \\
= & \tilde{u}_{n}-u_{n}+h\left[f\left(t_{n+1}, v_{n+1}, \tilde{u}_{n}\right)-f\left(t_{n+1}, v_{n+1}, u_{n}\right)\right] \\
& +h\left[f\left(t_{n+1}, v_{n+1}, u_{n}\right)-f\left(t_{n+1}, u_{n+1}, u_{n}\right)\right]+\delta_{n+1} .
\end{aligned}
$$

Setting

$$
\varepsilon_{n}:=v_{n}-u_{n},
$$

and taking the scalar product of both sides of (16) by $\varepsilon_{n+1}$, we obtain, using repeatedly the Schwarz inequality and (10), (11),

$$
\left\|\varepsilon_{n+1}\right\| \leq\left(1+h K_{2}\right)\left\|\tilde{u}_{n}-u_{n}\right\|+h K_{1}\left\|\varepsilon_{n+1}\right\|+\left\|\delta_{n+1}\right\|,
$$


and then, for $h K_{1}<1$ (which is only required when $K_{1}>0$ ),

$$
\left\|\varepsilon_{n+1}\right\| \leq \frac{1+h K_{2}}{1-h K_{1}}\left\|\tilde{u}_{n}-u_{n}\right\|+\frac{\left\|\delta_{n+1}\right\|}{1-h K_{1}} .
$$

Therefore, by (15),

$$
\left\|\tilde{u}_{n+1}-u_{n+1}\right\| \leq \frac{1+h K_{2}}{1-h K_{1}}\left\|\tilde{u}_{n}-u_{n}\right\|+\frac{\left\|\delta_{n+1}\right\|}{1-h K_{1}}+\left\|\sigma_{n+1}\right\| .
$$

Setting, for short,

$$
a_{n}:=\left\|\tilde{u}_{n}-u_{n}\right\|, \quad \rho:=\frac{1+h K_{2}}{1-h K_{1}}, \quad \tau:=\frac{1}{1-h K_{1}},
$$

(20) becomes

$$
a_{n+1} \leq \rho a_{n}+\tau\left\|\delta_{n+1}\right\|+\left\|\sigma_{n+1}\right\|, \quad n=0,1, \ldots, N-1,
$$

where $a_{0}:=\left\|\delta_{0}\right\|$. Then, inductively obtains

$$
a_{n} \leq \rho^{n}\left\|\delta_{0}\right\|+\tau \sum_{k=1}^{n} \rho^{n-k}\left\|\delta_{k}\right\|+\sum_{k=1}^{n} \rho^{n-k}\left\|\sigma_{k}\right\| .
$$

Recalling that $K_{1} \in R, K_{2}>0$, it is convenient to list separately two cases:

a) $K_{1}+K_{2} \leq 0$, then $0<\rho \leq 1$, and from (23)

$$
\max _{0 \leq n \leq N}\left\|\tilde{u}_{n}-u_{n}\right\| \leq\left\|\delta_{0}\right\|+\tau \sum_{k=1}^{N}\left\|\delta_{k}\right\|+\sum_{k=1}^{N}\left\|\sigma_{k}\right\|=: \phi_{h}(e),
$$

$\phi_{h}(e)$ being that introduced in $\S 2$;

b) $K_{1}+K_{2}>0$, then $\rho>1$, and

$$
\max _{0 \leq n \leq N}\left\|\tilde{u}_{n}-u_{n}\right\| \leq \rho^{N}\left(\left\|\delta_{0}\right\|+\tau \sum_{k=1}^{N}\left\|\delta_{k}\right\|+\sum_{k=1}^{N}\left\|\sigma_{k}\right\|\right)=: \phi_{h}(e) .
$$

Here

$$
\rho^{N}=\left(\frac{1+\frac{K_{2} T}{N}}{1-\frac{K_{1} T}{N}}\right)^{N} \leq c \exp \left\{\left(K_{1}+K_{2}\right) T\right\}
$$

$\left(\rho^{N} \sim \exp \left\{\left(K_{1}+K_{2}\right) T\right\}, N \rightarrow \infty\right)$ where the constant $c$ can be taken equal to 1 when $K_{1}>0$. 
Finally, the map $F_{h}$ (cf. $\left.\S 2\right)$ being a linear interpolant, we have

$$
\left\|F_{h}(x, e)-F_{h}(x, 0)\right\| \leq \max _{0 \leq n \leq N}\left\|\tilde{u}_{n}-u_{n}\right\| \leq \phi_{h}(e), h K_{1}<1
$$

where $x=u_{0}$, and $\|u\|=\|u\|_{Y}:=\sup _{0<t<T}\|u(t)\|$, which shows stability, according to $\S 2$ (such an estimate is referred to as "order stability" in [7]).

Remark 3.1 In the special case that $f(t, \cdot, v)$ is Lipschitz continuous in the classical sense with constant $K_{3}>0$, the error $\sigma_{n+1}$ in (14) due to the numerical solution of the first equation in (14), can be adsorbed in $\delta_{n+1}$. In fact, one may think that $\tilde{u}_{n+1}$ is the (unique) solution to

$$
z=\tilde{u}_{n}+h f\left(t_{n+1}, z, \tilde{u}_{n}\right)+h\left[f\left(t_{n+1}, v_{n+1}, \tilde{u}_{n}\right)-f\left(t_{n+1}, \tilde{u}_{n+1}, \tilde{u}_{n}\right)\right]+\delta_{n+1} .
$$

Note that here one should require that $\tilde{u}_{n} \in D_{t_{n}}$ for every $n \geq 1$, that is that the method followed to solve the first equation in (14) yields an approximate solution, $\tilde{u}_{n+1}$, in $D_{t_{n+1}}$. Comparing with the first equation in (14), we see that $\delta_{n+1}$ there is replaced by

$$
\delta_{n+1}^{*}=\delta_{n+1}+h\left[f\left(t_{n+1}, v_{n+1}, \tilde{u}_{n}\right)-f\left(t_{n+1}, \tilde{u}_{n+1}, \tilde{u}_{n}\right)\right],
$$

and hence a scheme like (14) is obtained with $\sigma_{n+1}=0$. Then,

$$
\left\|\delta_{n+1}^{*}\right\| \leq\left\|\delta_{n+1}\right\|+h K_{3}\left\|v_{n+1}-\tilde{u}_{n+1}\right\|=\left\|\delta_{n+1}\right\|+h K_{3}\left\|\sigma_{n+1}\right\|
$$

which shows that in the estimates (24)-(25) absorbing $\sigma_{n+1}$ in $\delta_{n+1}$ is advantageous provided that $\tau h K_{3}<1$, i.e. $\left(K_{1}+K_{3}\right) h<1$. When $K_{1}+K_{3}>0$, this is an actual condition on the time step. Note that, in any case, there is a contraction by a factor $\tau h K_{3} \sim h K_{3}$ as $h \rightarrow 0$.

The occurrence described above is typical of many systems of ordinary differential equations, while it is ruled out in case of partial differential equations (for which $f(t, \cdot, v)$ is a differential operator).

\subsection{Consistency of the semi-implicit Euler method.}

To prove consistency $(\S 2)$, we write

$$
u\left(t_{n+1}\right)=u\left(t_{n}\right)+h f\left(t_{n+1}, u\left(t_{n+1}\right), u\left(t_{n}\right)\right)+\omega_{n+1}(h),
$$

that is (14) with the perturbation vector $\hat{e}_{h}(x)=(\delta, \sigma)=\left(0, \omega_{1}(h), \ldots, \omega_{N}(h) ; 0, \ldots, 0\right)$, where $x=u_{0}$, cf. (7). Here $u(t)$ is the unique solution to $(9)$, with $u \in C^{1}[0, T]$ or $u \in A C[0, T], \dot{u} \in B V[0, T]$. Clearly, (7) is automatically satisfied by the uniform 
continuity of $u(t)$ on $[0, T], F_{h}\left(u_{0}, \hat{e}_{h}\left(u_{0}\right)\right)$ being the linear interpolant among the values $u\left(t_{n}\right), n=0,1, \ldots, N$. What remains to be proved is (8).

We have to show that $\phi_{h}\left(\hat{e}_{h}\left(u_{0}\right)\right) \rightarrow 0$ as $h \rightarrow 0$, that is equivalent to show that $\sum_{k=1}^{N}\left\|\omega_{k}(h)\right\| \rightarrow 0$, as $h \rightarrow 0$, for $u_{0}$ fixed (cf. (13) with $\delta_{0}=0, \sigma_{k}=0$ ). Now, from $(31)$

$$
\begin{gathered}
\left\|\omega_{k+1}(h)\right\|=\left\|u\left(t_{k+1}\right)-u\left(t_{k}\right)-h f\left(t_{k+1}, u\left(t_{k+1}\right), u\left(t_{k}\right)\right)\right\| \\
=\| \int_{t_{k}}^{t_{k+1}} \dot{u}(t) d t-h \dot{u}\left(t_{k+1}\right)-h\left[f\left(t_{k+1}, u\left(t_{k+1}\right), u\left(t_{k}\right)\right)-f\left(t_{k+1}, u\left(t_{k+1}\right), u\left(t_{k+1}\right)\right) \|\right. \\
\leq\left\|h \sum_{i=1}^{m_{k}} \beta_{k, i}\left[\dot{u}\left(\tau_{k, i}\right)-\dot{u}\left(t_{k+1}\right)\right]+w_{\varepsilon}\right\|+h K_{2}\left\|u\left(t_{k+1}\right)-u\left(t_{k}\right)\right\| .
\end{gathered}
$$

Here the fact that $u \in A C[0, T]$ has been used. Besides, as $\dot{u}(t)$ is Riemann integrable on $[0, T]$ (cf. [13, Ch.3]), for every $\varepsilon>0$, and for every $k, k=1,2, \ldots, N$, the nodes $\tau_{k, i}$, and the weights $\beta_{k, i}>0, i=1,2, \ldots, m_{k}$, with $\sum_{i=1}^{m_{k}} \beta_{k, i}=1$, exist so as to approximate the integral of $\dot{u}$ by the finite sum above, up to an error $w_{\varepsilon},\left\|w_{\varepsilon}\right\|<\varepsilon$. Then

$$
\left\|\omega_{k+1}(h)\right\| \leq h \sum_{i=1}^{m_{k}} \beta_{k, i}\left\|\dot{u}\left(\tau_{k, i}\right)-\dot{u}\left(t_{k+1}\right)\right\|+\varepsilon+h K_{2}\left\|u\left(t_{k+1}\right)-u\left(t_{k}\right)\right\| .
$$

Now, when $u^{\prime} \in C^{0}[0, T]$, we get

$$
\sum_{k=1}^{N}\left\|\omega_{k}(h)\right\| \leq h N\left[\operatorname{osc}(\dot{u} ; h)+K_{2} \operatorname{osc}(u ; h)\right],
$$

where $h N=T$, and

$$
\operatorname{osc}(u ; h):=\sup \left\{|| u\left(t_{1}\right)-u\left(t_{2}\right) \|: t_{1}, t_{2} \in[0, T],\left|t_{1}-t_{2}\right| \leq h\right\},
$$

is a certain definition of "oscillation" of stepsize $h$ (modulus of continuity). Hence $\phi_{h}\left(\hat{e}_{h}\left(u_{0}\right)\right)=o(1)$, being osc $(u ; h)=O(h)$ and $\operatorname{osc}(\dot{u} ; h)=o(1)$.

When $\dot{u} \in B V[0, T]$, we can estimate $\sum\left\|\omega_{k}(h)\right\|$ in a different way. In fact, choosing (as is permissible) $m_{k}$ and $\beta_{k, i}$ independent of $k$ in (33), and interchanging the sums (over $k$ and $i$ ), obtains, instead of (34),

$$
\sum_{k=1}^{N}\left\|\omega_{k}(h)\right\| \leq h V_{0}^{T}\left(u^{\prime}\right)+T K_{2} \operatorname{osc}(u ; h),
$$

$V_{0}^{T}$ denoting the variation on $[0, T]$. Therefore $\phi_{h}\left(\hat{e}_{h}\left(u_{0}\right)\right)=O(h)(\operatorname{as} \operatorname{osc}(u ; h)=$ $O(h), \dot{u}$ being bounded), and thus consistency is proved.

Remark 3.2 (order of consistency) If $\dot{u}$ is Lipschitz continuous in $[0, T]$, then the r.h.s of (34) is $O(h)$. If $\dot{u} \in B V[0, T] \cap C^{0}[0, T],(36)$ is in general a better estimate than (34), being always of $O(h)$, in view of the boundedness of $\dot{u}$. 


\subsection{Convergence estimates.}

From the results of $\S 3.1, \S 3.2$, convergence of the semi-implicit Euler method (cf. (5)) follows by a Lax-type equivalence theorem [7]. Following the proof of the theorem in [7], we obtain by (27)

$$
\begin{gathered}
\left\|\left|F_{h}\left(u_{0}, e\right)-u\right|\right\| \leq\left\|\left|F_{h}\left(u_{0}, e\right)-F_{h}\left(u_{0}, 0\right)\right|\right\|+\left\|\mid F_{h}\left(u_{0}, 0\right)-F_{h}\left(u_{0}, \hat{e}_{h}\left(u_{0}\right)\right)\right\| \| \\
+\left\|\left|F_{h}\left(u_{0}, \hat{e}_{h}\left(u_{0}\right)\right)-u \|\right| \leq \phi_{h}(e)+\phi_{h}\left(\hat{e}_{h}\left(u_{0}\right)\right)+\operatorname{osc}(u ; h),\right.
\end{gathered}
$$

where $\||u|\|=\|u\|_{Y}:=\sup _{0<t<T}\|u(t)\|$, since the oscillation term, osc $(u ; h)$, estimates the error made in the linear interpolation of the exact solution, $u$. Now, the fact that $\dot{u}$ is bounded (implied by either $u \in C^{1}[0, T]$, or by $\dot{u} \in B V[0, T]$ ) entails that $\operatorname{osc}(u ; h)=O(h)$. For convenience, we display the convergence estimates obtained from (37) and (24)-(25). Setting

$$
r_{N}:=\left\{\begin{array}{cl}
1, & \text { if } K_{1}+K_{2} \leq 0 \\
\rho^{N} \equiv\left(\frac{1+K_{2} T / N}{1-K_{1} T / N}\right)^{N}, & \text { if } K_{1}+K_{2}>0,
\end{array}\right.
$$

we have

$$
\begin{gathered}
\left\|\left|F_{h}\left(u_{0}, e\right)-u \|\right| \leq r_{N}\left\{\left\|\delta_{0}\right\|+\tau \sum_{k=1}^{N}\left\|\delta_{k}\right\|+\sum_{k=1}^{N}\left\|\sigma_{k}\right\|\right.\right. \\
\left.+\tau T\left(\operatorname{osc}\left(u^{\prime} ; h\right)+K_{2} \operatorname{osc}(u ; h)\right)\right\}+\operatorname{osc}(u ; h),
\end{gathered}
$$

when $\dot{u} \in C^{0}[0, T]$, and

$$
\begin{gathered}
\left\|\mid F_{h}\left(u_{0}, e\right)-u\right\| \leq r_{N}\left\{\left\|\delta_{0}\right\|+\tau \sum_{k=1}^{N}\left\|\delta_{k}\right\|+\sum_{k=1}^{N}\left\|\sigma_{k}\right\|\right. \\
\left.+\tau\left(h V_{0}^{T}\left(u^{\prime}\right)+T K_{2} \operatorname{osc}(u ; h)\right)\right\}+\operatorname{osc}(u ; h),
\end{gathered}
$$

when $\dot{u} \in B V[0, T]$.

From the computational viewpoint, in (39), (40) it is reasonable to take $e=$ $\left(\delta_{0}, 0, \ldots, 0 ; \sigma_{1}, \ldots, \sigma_{N}\right)$ where $\delta_{0}$ is the error on the initial value and $\sigma_{k}$ represents the overall error made in the numerical solution of the $k$-th equation in (14). Such an error takes into account, typically, round-off errors and the error inherent to the method itself used to solve the $k$-th equation in (14). In case of partial differential equations, for instance, the latter takes into account, in turn, the error in the boundary data (if any) and the truncation error with respect to the space variables. In the theory above, perturbation terms $\delta_{k} \neq 0(k=1,2, \ldots, N)$ have been included in studying stability. In fact they appear, in a natural way, as local truncation errors, $\omega_{k}(h)$ (cf. (31), §3.2). 
Therefore, (40), as well as (39) when $\dot{u}$ is Lipschitz continuous in $[0, T]$, yields the following qualitative convergence estimate:

$$
\left\|\mid F_{h}\left(u_{0}, e\right)-u\right\|=O\left(\left\|\delta_{0}\right\|\right)+O\left(\sum_{k=1}^{N}\left\|\sigma_{k}\right\|\right)+O(h),
$$

since $r_{N} \rightarrow \exp \left\{\left(K_{1}+K_{2}\right) T\right\}$ and $\tau \rightarrow 1$ as $h \rightarrow 0$ (cf. (21). The practical use of the method rests on having at hand accurate algorithms to solve the first equation in (14) (with $\delta_{n+1}=0$ ), since, roughly speaking, one should guarantee that $h^{-1} \max _{1 \leq k \leq N}\left\|\sigma_{k}\right\|$ be small when $h$ is small.

Remark 3.3 Everything done in this section holds in case of a complex Hilbert space, just taking the real part on the 1.h.s. of (10) and similarly after then.

Remark 3.4 All results obtained in this section hold when $\mathcal{H}$ is replaced by a general (real or complex) Banach space $\mathcal{B}$, provided that $f(t, u, \cdot)$ is Lipschitz continuous as before, $\mathcal{R}(I-h f(t, \cdot, v))=\mathcal{B}$ for $h>0$ sufficiently small, and $f(t, \cdot, v)$ satisfies, for every fixed $t \in(0, T]$ and $v \in D$, the condition

$$
\left\|u_{1}-u_{2}-h\left[f\left(t, u_{1}, v\right)-f\left(t, u_{2}, v\right)\right]\right\| \geq\left(1-h K_{1}\right)\left\|u_{1}-u_{2}\right\|, \quad \forall u_{1}, u_{2} \in D_{t} .
$$

This property is equivalent to that in (10) above when the space is a Hilbert space [20]. and has beeen extensively used in the numerical treatment of ordinary differ-

ential systems, (cf. $[9,29]$, e.g.). When $K_{1}=0$, the operator $f(t, \cdot, v)$ is termed "dissipative"; in general, in (42) $K_{1}$ can be positive, negative, or zero. The straightforward modifications in $\S 3.1$ are left to the reader.

Remark 3.5 Note that the analysis developed in Section 3 embodies the case of the fully implicit Euler method. In fact, if $K_{1} \in R$ denotes the one-sided Lipschitz constant of $p(t, u)$ with respect to $u$, where $p(t, u):=f(t, u, u)$, with $\mathcal{R}(I-h p(t, \cdot))=$ $\mathcal{H}$ for $h>0$ sufficiently small, the theory of Section 3 can be applied with $K_{2}=0$. For an application of the theory of [7] to the fully implicit Euler method with $K_{1}=0$, see [31]. We stress that the case $K_{1}>0$ is also included, which occurrence seems to be not recorded in the literature for the infinite-dimensional case.

\section{Examples.}

In this Section, we present some applications of the semi-implicit Euler method, in both the finite and the infinite-dimensional case. The computational advantages in using such a method will be pointed out. Below, letters denoting $m$-dimensional vectors will be boldfaced, for clarity. 
A) Systems of ordinary differential equations.

Consider equation (9) with $\mathcal{H}=R^{m}$, that is an $m$-dimensional system of ordinary differential equations (ODEs). Assume, moreover, that all hypotheses accompanying equation (9) in Section 3 are satisfied. Note that such hypotheses are classical in the context of the numerical treatment of systems of ODEs (cf. [9], e.g.). Our approach is closely related to the so-called "backward Euler multirate method", used to decouple system (9) into stationary sub-systems, in view of parallel implementation, cf. [28, 29]. The latter represents an approach alternative to the "waveform relaxation (WR) method" [11] used to solve very large, possibly stiff systems. An advantage of such an approach with respect to the WR method is that no iteration is required in the continuous time domain. Indeed, the convergence of such an iteration can be very slow, and memory occupacy for storing the waveforms may be large.

An important example treated in [29] is the "decoupled implicit Euler method" for $\dot{u}=F(t, u) \equiv f(t, u, u), u(0)=u_{0}$, where

$$
f(t, u, v)=\left(F_{1}\left(t, u_{1}, v_{2}, \ldots, v_{r}\right), \ldots, F_{r}\left(t, v_{1}, \ldots v_{r-1}, u_{r}\right)\right) .
$$

Here $F_{k}, u_{k}$ are $m_{k}$-dimensional vectors, and $v_{j}$ is $m_{j}$-dimensional; $\sum_{k=1}^{r} m_{k}=m$. In this way one is facing, at each time step, $r$ independent nonlinear algebraic systems, which can be solved concurrently (cf. (14) above, with $\delta_{n+1}=0$ ).

As shown in [29], when $F$ is sufficiently smooth, extrapolation can be used to obtain an higher order of integration accuracy. In the opposite direction, our analysis also allows $f(t, \cdot, v)$ to be discontinuous. This feature is essential in solving evolutionary partial differential equations and systems.

B) A single reaction-diffusion equation in $L^{2}(\Omega)$.

Consider the problem

$$
\begin{gathered}
w_{t}=a \Delta w+g(t, x, w), \text { for } x \in \Omega \subset R^{d}, \Omega \text { open smooth bounded, } 0<t \leq T, \\
w=w_{1}(x, t) \text { on } \partial \Omega ; w(x, 0)=w_{0}(x), x \in \Omega,
\end{gathered}
$$

where $\Delta$ denotes the Laplace operator in $R^{d}, g$ is globally Lipschitz-continuous with respect to $w$, uniformly in $x \in \Omega$ and $t \in(0, T]$. Moreover, assume that for every $t \in(0, T], g(t, \cdot, 0) \in L^{2}(\Omega)$ and $w_{0}$ and $w_{1}$ are suitable functions. The problem $(44)$ can be recast into the form of equation (1) with $\mathcal{H}=L^{2}(\Omega), A(t, \cdot) \equiv A(\cdot)=a \Delta$, $D_{t}=\left\{u \in H^{2}(\Omega): u=w_{1}\right.$ on $\left.\partial \Omega\right\}$, and $B(t, \cdot): u(t)=w(\cdot, t) \mapsto g(t, \cdot, w(\cdot, t))$, $D=L^{2}(\Omega)$.

The hypotheses on $g$ above imply that $g(t, \cdot, w(\cdot, t)) \in L^{2}(\Omega)$, and that $B(t, \cdot)$ is Lipschitz-continuous as a map from $L^{2}(\Omega)$ into $L^{2}(\Omega)$ (see [4], e.g.). On the other hand, $A$ is a maximal dissipative operator, as known (cf. [5]). 
We assume, further, that additional conditions are fulfilled ensuring existence of $C^{1}[0, T]$ solutions; this is the case, for instance, when $w_{1} \equiv 0$ (and hence $D_{t} \equiv D(A)=$ $\left.H^{2}(\Omega) \cap H_{0}^{1}(\Omega)\right), B$ is a $C^{1}$-function of its arguments, and $u_{0}=w_{0}(\cdot) \in D(A)$, cf. [23, Thm.1.5, p.187]. Unfortunately, the substitution operator $B(t, \cdot)$ is differentiable if and only if the scalar function $g(t, x, w)$ in (44) is linear in $w$ (cf. [15, Thm. 20.1, p. 419]). This restriction, however, can be overcome when $g$ is Lipschitz continuous in both $t$ and $w$, uniformly with respect to $x \in \Omega$. In fact, the substitution operator turns out to be Lipschitz continuous in both $t$ and $u$, which ensures the existence of a "strong" solution in the sense of [23, Thm.1.6, p.189]. Such a strong solution is actually continuously differentiable in $[0, T]$, which fact can be seen as follows. One considers first the linear inhomogeneous problem $\dot{u}=A u+b(t), t>0, u(0)=u_{0} \in$ $D(A)$, with $b$ Lipschitz continuous in $[0, T]$. It is known [12, Remark 9.8, p.129] that there is a unique solution which is continuously differentiable in $(0, T]$. By standard arguments of linear semigroups theory one then shows that $\dot{u}$ can be continued up to $t=0$ from the right. Finally, combining this argument with the proof of Theorem 1.6 of [23, p.189], extension can be made to the semilinear problem we are considering.

We observe that the restriction that $g$ be globally Lipschitz-continuous with respect to $w$ can be removed whenever a priori estimates on the solution, say $|w(x, t)| \leq$ $M$ for all $x \in \bar{\Omega}$ and $t \in[0, T]$, are available, and $g$ is Lipschitz-continuous in $[-M, M]$ uniformly in $x \in \bar{\Omega}$ and $t \in(0, T]$. In this case, in fact, problem (44) can be replaced by a similar one where $g$ is replaced by $g^{*}(t, x, w)=g(t, x, w)$ in $(0, T] \times \Omega \times[-M, M]$ and $g^{*}(t, x, w)=g(t, x, M)$ for $w>M, g^{*}(t, x, w)=g(t, x,-M)$ for $w<-M$, cf. [14].

The computational advantages of the method are clear. At each time step, the semi-implicit Euler method applied to problem (44) requires solving the $d$-dimensional linear inhomogeneous Helmholtz equation

$$
\begin{gathered}
v_{n+1}(x)=\tilde{u}_{n}(x)+h a \Delta v_{n+1}(x)+h g\left(t_{n+1}, x, \tilde{u}_{n}(x)\right), x \in \Omega, \\
v_{n+1}(x)=w_{1}\left(x, t_{n+1}\right), x \in \partial \Omega,
\end{gathered}
$$

(cf. (14) with $\delta_{n+1}=0$ ). A unique solution, $v_{n+1}$, to (45) does exist as $a \Delta$ is a maximal dissipative operator from $\left\{u \in H^{2}(\Omega): u(x)=w_{1}\left(x, t_{n+1}\right)\right.$ on $\left.\partial \Omega\right\}$ in $L^{2}(\Omega)$. The approximate solution to $(45)$, say $\tilde{u}_{n+1}(x)$, should be seeked in $D=L^{2}(\Omega)$, cf. (14). The equation in (45) being linear, its numerical treatment can be based on a variety of methods, besides the widely used finite difference and finite element methods. For instance, when $\Omega$ can be decomposed into rectangular subdomains, equations like (45) (as well as similar linear elliptic systems, cf. Example (D) below) can be treated by spectral collocation methods. These methods are known to possess an extremely high convergence rate, when the solution is smooth, cf. [25]. When the spatial dimension $d$ is comparatively large, one might apply probabilistic methods. We 
stress, incidentally, that in this approach parallelization is trivial, since the solution at every point $x \in \Omega$ is evaluated as an average over Brownian trajectories which can be generated independently (and hence on several different processors), cf. [21], e.g.. The direct application of such methods to the nonlinear evolution equation (44) is not straightforward, cf. [27].

C) A single semilinear parabolic equation in $C^{0}(\bar{\Omega})$.

The following example shows an application in a Banach space which is not a Hilbert space (cf. Remark 3.4):

$$
\begin{gathered}
w_{t}=L w+g(t, x, w), x \in \Omega \subset R^{d}, 0<t \leq T, \\
L u:=a_{i j}(x) u_{x_{i} x_{j}}+b_{i}(x) u_{x_{i}}-c(x) u, \\
w(x, t) \equiv 0, x \in \partial \Omega, 0<t \leq T ; \quad w(x, 0)=w_{0}(x), \quad x \in \Omega .
\end{gathered}
$$

Here, $a_{i j}, b_{i}, c \in C^{0}(\bar{\Omega}), c(x) \geq 0$ in $\Omega$, and $L$ is strictly elliptic, i.e. there is $\theta>0$ such that $a_{i j} \xi_{i} \xi_{j} \geq \theta|\xi|^{2}$ for every $d$-vector $\xi$ and $x$ in $\Omega ; g$ is Lipschitz-continuous in $w$ uniformly in $(x, t) \in \Omega \times(0, T]$, and $g(t, x, 0)=0$ for each pair $(x, t) \in \partial \Omega \times(0, T]$. In this case, the parabolic equation in (46) is of type (1) with $\mathcal{H}=C_{0}^{0}(\bar{\Omega})$ endowed with the sup-norm, $A(t, \cdot) \equiv L, D_{t} \equiv D(L)=\left\{u \in W^{2, p}(\Omega) \forall p<\infty: L u \in C^{0}(\bar{\Omega}), u \equiv\right.$ 0 on $\partial \Omega\}$, and $B(t, \cdot): u(t)=w(\cdot, t) \mapsto g(t, \cdot, w(\cdot, t)), D=C_{0}^{0}(\bar{\Omega})$. Besides, assume that $g \in C^{1}([0, T] \times \Omega \times R)$. Then $B \in C^{1}\left([0, T] \times C_{0}^{0}(\bar{\Omega}) ; C_{0}^{0}(\bar{\Omega})\right)($ cf. $[32$, p.167]) and thus, if $w_{0} \in D(L)$ by Theorem 1.5, p.187 of [23] the abstract problem (1) corresponding to $(46)$ has a unique $C^{1}\left([0, T] ; C_{0}^{0}(\bar{\Omega})\right)$ solution. Therefore the semiimplicit Euler method can be applied, and the computational advantage of facing a linear elliptic problem at each time step again follows.

D) Systems of reaction-diffusion equations.

Consider the system

$$
\begin{gathered}
w_{t}=M \Delta w+g(t, x, w), \quad x \in \Omega \subset R^{d}, 0<t \leq T, \\
w(x, 0)=w_{0}(x), x \in \Omega ; w=w_{1}(x, t) \text { on } \partial \Omega,
\end{gathered}
$$

for the $m$-dimensional vector $w$, where $M$ is a diagonal constant positive definite matrix. Here, $g(t, x, \cdot)$ is supposed to be a Lipschitz-continuous function from $R^{m}$ into $R^{m}$, uniformly in $(t, x)$, and $g_{i}(t, \cdot, 0) \in L^{2}(\Omega), i=1,2, \ldots, m$. To cast the problem into the abstract form (1), we choose $\mathcal{H}=\left(L^{2}(\Omega)\right)^{m}, A(t, \cdot)=M \Delta, B(t, \cdot): u(t)=$ $w(\cdot, t) \mapsto g(t, \cdot, w(\cdot, t)), D_{t}=\left\{u \in\left(H^{2}(\Omega)\right)^{m}: u_{i}=w_{1, i}\right.$ on $\left.\partial \Omega, i=1,2, \ldots, m\right\}$, $D=\mathcal{H}$. As in Example (B), it follows that $g(t, \cdot, w(\cdot, t)) \in \mathcal{H}$, and $B(t, \cdot)$ is Lipschitzcontinuous from $\mathcal{H}$ into $\mathcal{H}$. Moreover, we assume further that additional conditions are satisfied to ensure existence of $C^{1}[0, T]$ solutions. Similarly to Example (B), for 
instance, this is the case when $g$ is Lipschitz continuous in both $t$ and $w$ uniformly in $x \in \Omega$.

When the semi-implicit Euler method is applied to (47), one has to solve numerically $m$ linear (scalar) elliptic (Helmholtz) uncoupled equations at each time step, i.e.

$$
\begin{gathered}
v_{i, n+1}(x)=\tilde{u}_{i, n}(x)+h M_{i} \Delta v_{i, n+1}(x)+h g_{i}\left(t_{n+1}, x, \tilde{u}_{n}(x)\right), \\
v_{i, n+1}(x)=w_{1, i}\left(x, t_{n+1}\right) \text { on } \partial \Omega, \quad i=1,2, \ldots, m .
\end{gathered}
$$

Note that, in this case, besides linearization, decoupling takes place of the $m$ dimensional system into $m$ independent Helmholtz problems, which fact allows for a trivial parallel implementation. In particular, one may consider adopting different methods for each of the $m$ components, e.g. when the $M_{i}$ 's have very different size. This suggests also the following

Remark 4.1 The semi-implicit Euler method can be applied to systems of semilinear parabolic equations like those in (47) with $M=M_{1} \oplus M_{2} \oplus \ldots \oplus M_{k}$, where $M_{j}$ is an $m_{j} \times m_{j}$ constant positive definite matrix, $\sum_{j=1}^{k} m_{j}=m$. In this case, the method reduces to the solution of $k$ linear elliptic uncoupled subsystems, at each time step.

E) An integro-differential parabolic equation.

Consider

$$
\begin{gathered}
w_{t}=a \Delta w+\int_{\Omega} K(x, \xi) w(\xi, t) d \xi, x \in \Omega \subset R^{d}, 0<t \leq T, \\
w=w_{1} \text { on } \partial \Omega ; w(x, 0)=w_{0}(x), x \in \Omega,
\end{gathered}
$$

where $a>0$ and $K(\cdot, \cdot) \in L^{2}(\Omega \times \Omega)$. Again, equation (49) can be cast into the abstract form (1) with $\mathcal{H}=L^{2}(\Omega), A(t, \cdot) \equiv A(\cdot)=a \Delta, D_{t} \equiv D(A)=\left\{u \in H^{2}(\Omega)\right.$ : $u=w_{1}$ on $\left.\partial \Omega\right\}$, and $B(t, \cdot) \equiv B(\cdot): u(t)=w(\cdot, t) \mapsto \int_{\Omega} K(\cdot, \xi) w(\cdot, \xi) d \xi, D=L^{2}(\Omega)$. Note that the integral operator $B(t, \cdot)$ is linear bounded from $L^{2}(\Omega)$ into $L^{2}(\Omega)$ (since $K \in L^{2}(\Omega \times \Omega)$ ) and hence has Lipschitz constant $K_{2}=\|K\|_{L^{2}(\Omega \times \Omega)}$, cf. (11). Existence of solutions $u \in C^{1}\left([0, T] ; L^{2}(\Omega)\right)$ is guaranteed, for instance, when $w_{1} \equiv 0$ by Theorem 1.5, p.187 of [23], as $B(t, \cdot) \in C^{1}\left(L^{2}(\Omega) ; L^{2}(\Omega)\right)$. Therefore the semiimplicit Euler method can be applied, and requires solving the linear inhomogeneous elliptic (Helmholtz) equation

$$
\begin{gathered}
v_{n+1}(x)=\tilde{u}_{n}(x)+h a \Delta v_{n+1}(x)+h \int_{\Omega} K(x, \xi) \tilde{u}_{n}(\xi) d \xi, x \in \Omega, \\
v_{n+1}(x)=w_{1}\left(x, t_{n+1}\right), x \in \partial \Omega,
\end{gathered}
$$

at each time step. 
If, in addition, the integral operator in (49) is dissipative on $L^{2}(\Omega)$ (e.g., when the kernel $K(x, \xi)$ is symmetric negative semi-definite Hilbert-Schmidt [4, Example 7.3.1, p.234]), the fully implicit Euler method is also applicable (cf. 3.5). In fact, the whole operator $A(\cdot)+B(\cdot)$ is dissipative, in this case (cf. [19, 26]). This method exhibits better stability properties, since the "propagation factor" in $(26), \rho^{N}$, is replaced by 1 , but one must face, after space discretization, the solution of full high-dimensional linear systems. Following the semi-implicit Euler method, instead, one has to solve merely band structured (using finite differences), or sparse systems (finite elements), since only the differential operator is discretized at each time-step. Therefore, the latter approach requires handling only local operators versus integral operators.

Remark 4.2 We chose the linear scalar equation (49) for simplicity. All could be done for more general nonlinear reaction-diffusion systems like

$$
w_{t}=M \Delta w+g(t, x, w)+\int_{\Omega} K(t, x, \xi, w(\xi, t)) d \xi,
$$

for the $m$-vector $w$, with suitable initial-boundary data. Such problem generalizes all the other examples described above. Clearly, in discretizing numerically equation (51) by the semi-implicit Euler method all the advantages pointed out above (linearization, decoupling, local versus nonlocal structure) are obtained at the same time. It is worth noting that in the block-diagonal matrix $M$ in (51) some blocks are allowed to vanish. This means that in the integro-differential system (51), some subsystems may reduce to ordinary (integro-differential) systems. This happens in several models, e.g. concerning competing species dynamics (cf. [18]).

F) A semilinear hyperbolic equation.

The problem

$$
\begin{gathered}
w_{t t}=a \Delta w+g(t, x, w), \text { in } \Omega \times(0, T], \\
w(x, 0)=w_{0}(x), w_{t}(x, 0)=w_{1}(x), x \in \Omega ; w(x, t)=0, x \in \partial \Omega, t \in(0, T],
\end{gathered}
$$

can be written as as an abstract evolutionary problem for the 2-vector $u(t)=\left(u_{1}(t), u_{2}(t)\right)$ $:=\left(w(\cdot, t), w_{t}(\cdot, t)\right)$

$$
\dot{u}=A(u)+B(t, u), \quad t \in(0, T] ; u(0)=\left(w_{0}(\cdot), w_{1}(\cdot)\right),
$$

where

$$
A:=\left(\begin{array}{cc}
0 & I \\
a \Delta & 0
\end{array}\right), B(t, \cdot):\left(\left(u_{1}(t), u_{2}(t)\right) \mapsto(0, g(t, \cdot, w(\cdot, t))) .\right.
$$

Here, $\mathcal{H}=H_{0}^{1}(\Omega) \times L^{2}(\Omega), D_{t} \equiv D(A)=\left(H^{2}(\Omega) \cap H_{0}^{1}(\Omega)\right) \times H_{0}^{1}(\Omega), D=\mathcal{H}$, and $g$ satisfies the same hypotheses of Example (B). As is known [5], in this case $A$ is 
a maximal dissipative operator, and $B(t, \cdot)$ is Lipschitz continuous. Moreover, we assume that suitable assumptions are made to ensure existence of $C^{1}[0, T]$ solutions. Hence, the semi-implicit Euler method can be applied (see [24] for the fully implicit), yielding the system

$$
\begin{aligned}
& \left\{\begin{array}{l}
v_{1, n+1}(x)=\tilde{u}_{1, n}(x)+h v_{2, n+1}(x) \\
v_{2, n+1}(x)=\tilde{u}_{2, n}(x)+h a \Delta v_{1, n+1}(x)+h g\left(t_{n+1}, x, \tilde{u}_{1, n+1}(x)\right), \quad x \in \Omega,
\end{array}\right. \\
& v_{1, n+1}(x)=0, v_{2, n+1}(x)=0, x \in \partial \Omega,
\end{aligned}
$$

for $\left(v_{1, n+1}(x), v_{2, n+1}(x)\right)$, at each time step (cf. 14) with $\delta_{n+1}=0$ ). Such a system is equivalent to

$$
\left\{\begin{array}{l}
v_{1, n+1}(x)=h^{2} a \Delta v_{1, n+1}(x)+\tilde{u}_{1, n}(x)+h \tilde{u}_{2, n}(x)+h^{2} g\left(t_{n+1}, x, \tilde{u}_{1, n}(x)\right) \\
h v_{2, n+1}(x)=v_{1, n+1}(x)-\tilde{u}_{1, n}(x),
\end{array}\right.
$$

with the same data on $\partial \Omega$. Therefore, one should solve a linear inhomogeneous Helmholtz equation at each time step.

An interesting example of a semilinear hyperbolic equation with globally Lipschitz nonlinear term is given by the Sine-Gordon equation, $w_{t t}=\Delta w-\lambda \sin (w), \lambda>0$.

For hyperbolic semilinear systems for the $m$-vector $w$, such as

$$
w_{t t}=M \Delta w+g(t, x, w), M \text { diagonal } m \times m \text { matrix }
$$

as well as for the corresponding integro-differential systems (cf. equation (51) for the parabolic case), with initial-boundary data as in (52), the semi-implicit Euler method reduces essentially to the numerical solution of $m$ independent Helmholtz equations, at each time step.

\section{$5 \quad$ Final observations.}

In closing, some remarks are in order.

Remark 5.1 The assumption that $f(t, u, \cdot)$ be globally Lipschitz-continuous may be not restrictive in practice, when equation (9) is equivalent to an ordinary or to a partial differential equation or system. For several reaction-diffusion systems like those in (47) with $g(t, x, \cdot)$ locally Lipschitz-continuous in $R^{m}$, for instance, a priori estimates on $w$ are known (cf. [18], e.g.). In this case, the original problem can be transformed into an equivalent problem with the required property (cf. [14]). 
Remark 5.2 Examples (B)-(F) above are semilinear, but this was done merely for the purpose of illustration. The semi-implicit method analyzed in Section 3 can actually be applied to fully nonlinear equations. For instance, the method is still convenient when $f(t, u, u)$ has the additive structure, $A(t, u)+B(t, u)$, but $A$ is nonlinear, and the stationary equations, $v_{n+1}=\tilde{u}_{n}+h A\left(t_{n+1}, v_{n+1}\right)+h B\left(t_{n+1}, \tilde{u}_{n}\right)$, do present computational advantages.

In the remark below, the following results, valid in an arbitrary Banach space, will be used:

(i) If $A$ is maximal dissipative, and $B$ is everywhere defined, dissipative and Lipschitzcontinuous (and thus maximal dissipative), then $A+B$ is maximal dissipative;

(ii) If $A$ is maximal dissipative, $B$ is everywhere defined and Lipschitz-continuous, and $A+B$ is dissipative, then $A+B$ is maximal dissipative.

Proof. The part $(i)$ is proved, e.g., in [1], even for $B$ everywhere defined, dissipative and merely continuous. As for (ii), it suffices to show that

$$
u=h(A+B)(u)+b
$$

has a solution for every $b$ in $\mathcal{H}$ and for $h>0$ sufficiently small. In fact, the same will be true for every $h>0$ since $A+B$ is dissipative.

Consider the sequence of equations

$$
u^{n+1}=h A\left(u^{n+1}\right)+h B\left(u^{n}\right)+b, \quad n=0,1,2, \ldots
$$

$u^{0} \in \mathcal{H}$ arbitrary. They have a (unique) solution, $u^{n+1} \in D(A)$, since $A$ is maximal dissipative. It is easily seen by the same technique used in $\S 3.1$ to prove stability, that $\left\{u^{n}\right\}$ is a Cauchy sequence, and thus $u^{n} \rightarrow u^{*} \in \mathcal{H}$. Consider then

$$
z=h A(z)+h B\left(u^{*}\right)+b
$$

It has a (unique) solution, $z \in D(A)$, for the same reason above. Again, by a procedure similar to that in $\S 3.1$, one can show subtracting (60) from (59) that $u^{n} \rightarrow z$. Therefore $z=u^{*}$. Q.E.D.

Remark 5.3 When $K_{1}+K_{2} \leq 0$ (see (10), (11)), and thus the operator $p(t, u)=$ $A(t, u)+B(t, u)$ in (1) is globally dissipative in $u$, and hence maximal by (ii), the semi-implicit method has the same stability properties as the fully implicit one, since the propagation factor is $r_{N}=1$ in both cases (cf. (11) and Remark 3.5). When $K_{1}+K_{2}>0$, but $K_{1} \leq 0$ and $B(t, \cdot)$ is also dissipative (and then $p(t, \cdot)$ is maximal dissipative by $(i)$ ), the fully implicit method exhibits better stability properties. One 
should notice, however, that the semi-implicit approach still retains the computational advantages emphasized in the Examples above. Indeed, in Examples (B)-(F) above we have $K_{1}+K_{2}=K_{2}>0$, with $A(t, \cdot)$ dissipative, but $B(t, \cdot)$ may also be dissipative (for instance, this happens in Example (B) when $g$ is a decreasing function of $w$, see [4, Ch.3], besides being Lipschitz-continuous in $w$ ). A case when $K_{1}+K_{2} \leq 0$, instead, occurs in Example $(\mathrm{C})$ with $c(x) \geq c_{0}>0$, and the Lipschitz constant of $g$ in (46) $K_{2} \leq c_{0}$. In fact, the operator $L$ is now strongly dissipative with $K_{1}=-c_{0}$.

Acknowledgements. This work has been supported by the MURST Numerical Analysis funds (40\%-funds), and by the "Istituto Nazionale di Alta Matematica F. Severi".

\section{References}

[1] V. BARBU, Nonlinear semigroups and differential equations in Banach spaces, Noordhoff, Leyden, 1976.

[2] I. Babuska, H. Prager and E. Vitasek, Numerical Processes in Differential Equations, John Wiley, New York, 1966.

[3] A. Belleni-Morante, Applied semigroups and evolution equations, Clarendon Press, Oxford, 1979.

[4] R.K. Bose AND M.C. JoshI, Some topics in nonlinear functional analysis, Wiley Eastern Limited, New Dehli, 1985.

[5] H. Brezis, Analyse fonctionelle - Théorie et applications, Masson, Paris, 1983.

[6] V. Casulu, Semi-implicit finite difference methods for the two-dimensional shallow water equations, J. Comput. Phys. 86 (1990), 56-74.

[7] B. Chartres and R. Stepleman, A general theory of convergence for numerical methods, SIAM J. Numer. Anal. 9 (1972), 476-492.

[8] B. Chartres and R. Stepleman, Actual order of convergence of Runge-Kutta methods for differential equations with discontinuities, SIAM J. Numer. Anal. 11 (1974), 1193-1206.

[9] K. DEKKER AND J.G. VERWER, Stability of Runge-Kutta methods for stiff nonlinear differential equations, Elsevier Science Publishers, Amsterdam, 1984. 
[10] L.C. Evans, Nonlinear evolution equations in an arbitrary Banach space, Israel J. Math. 26 (1977), 1-42.

[11] C.W. GEAR AND FEn-Lien JUANG, The speed of waveform methods for ODEs, in: R. Spigler ed., Mathematics and its Applications: West Series, vol. 56, KLUWER, Dordrecht, 1991, pp. 37-48.

[12] A. Haraux, Linear Semigroups in Banach Spaces, in: H. Brezis, M.G. Crandall and F. Kappel eds., Semigroups, theory and applications (vol. II), Longman, Harlow (UK), 1986.

[13] E. Hille AND R.S. PhilliPs, Functional analysis and semi-groups, Amer. Math. Soc. Colloq. Publ. vol.31, Providence (R.I.), 1957.

[14] C. Johnson, S. Larsson, V. Thomée and L.B. Wahlbin, Error estimates for spatially discrete approximations of semilinear parabolic equations with nonsmooth initial data, Math. Comp. 49 (1987), 331-357.

[15] M.A. KRAsnoselskit ET AL., Integral operators in spaces of summable functions, Noordhoff, Leyden, 1976.

[16] V. Lakshmikantham and S. Leela, Differential and Integral Inequalities, vol.I, Academic Press, New York and London, 1969.

[17] P.D. LAX AND R. RICHTMEYER, Survey of the stability of linear difference equations, Comm. Pure App. Math. 9 (1956), 267-293.

[18] A.W. LEUng, Systems of nonlinear partial differential equations, KLUWER, Dordrecht, 1989.

[19] G. LIPPOLD, Error estimates and step size control for the approximate solution of a first order evolution equation, RAIRO Model. Math. Anal. Numer. 25 (1991), 110-128.

[20] R.H. MaRTIN, Nonlinear operators and differential equations in Banach spaces, John Wiley and Sons, New York, 1976.

[21] M. Mascagni, High dimensional numerical integration and massively parallel computing, Contemporary Mathematics 115 (1991), 53-73.

[22] J. NECAs, Application of Rothe's method to abstract parabolic equations, Czech. Math. J. 24 (1974), 496-500. 
[23] A. PAZY, Semigroups of linear operators and applications to partial differential equations, Springer-Verlag, New York, 1983.

[24] M. Pultar, Solution of abstract hyperbolic equations by Rothe method, Aplikace Matematiky 29 (1984), 23-39.

[25] A. QuARTERoni And E. ZAMPIERI, Finite element preconditioning for Legendre spectral collocation approximations to elliptic equations and systems, SIAM J. Numer. Anal. 29 (1992), 917-936.

[26] K. REKTORIS, Numerical and theoretical treating of evolution problems by the method of discretization in time, in: Equadiff 6, Proceedings (Brno, 1985), J. Vosmanský and M. Zlámal eds., Lecture Notes in Mathematics vol. 1192, SpringerVerlag, Berlin, 1986, pp. 70-84.

[27] A.S. Sherman and C.S. Peskin, A Monte Carlo method for scalar reactiondiffusion equations, SIAM J. Sci. Stat. Comput. 7 (1986), 1360-1372.

[28] J. SAND And S. Skelboe, Stability of backward Euler multirate methods and convergence of waveform relaxation, BIT 32 (1992), 350-366.

[29] S. Skelboe, Methods of parallel integration of stiff systems of ODEs, BIT 32 (1992), 689-701.

[30] V. Thomée, Galerkin Finite Element Methods for Parabolic Problems, Lecture Notes in Mathematics vol. 1054, Springer-Verlag, Berlin, 1984.

[31] M. Vianello and R. Zanovello, On some connections between wellposedness and general properties of numerical methods, ZAMM (1994), in press.

[32] N. Ya. Vilenkin ET AL., Functional Analysis, Wolters-Noordhoff, Groningen, 1972.

[33] E. ZeIDler, Nonlinear Functional Analysis and its Applications II/B: Nonlinear Monotone Operators, Springer-Verlag, New York, 1990. 